Bull. Austral. Math. Soc.

05B $15,05 \mathrm{C} 50,05 \mathrm{C} 70,05 \mathrm{E} 35,15 \mathrm{~A} 15,15 \mathrm{~A} 51$

VOL. 59 (1999) [169-170]

\title{
Permanents, matchings and Latin rectangles
}

\author{
IAN M. WANLESS
}

This thesis investigates three fundamentally related combinatorial objects: regular bipartite graphs, Latin rectangles and $(0,1)$-matrices with all line sums equal. The links between these objects are exploited to give some strong results on problems which had seemed intractable when considered in isolation. A central question, phrased in three ways, is the following:

(1) Which $k$-regular bipartite graphs on $2 n$ vertices contain the most perfect matchings?

(2) Which $(n-k) \times n$ Latin rectangles have the most extensions to $(n-k+1)$ $\times n$ Latin rectangles?

(3) Which square $(0,1)$-matrices of order $n$, with precisely $k$ ones in each row and column, achieve the maximum permanent?

Items (1) to (3) will be referred to below as the maximising problem. The most general previous result was due to Brègman [1], who solved the case when $k$ divides $n$. We essentially settle the complementary case (that is, when $n-k$ divides $n$ ) which relates to a conjecture of Godsil and McKay [3]. We also make substantial progress on the general case, which is an open question listed in Minc's survey article on permanents [6]. In fact that catalogue contains three problems (numbers 4,8 and 12) which we partly solve and five conjectures (numbers 12, 21, 22, 25 and 26) which we disprove. (Conjectures 25 and 26 were previously known to fail, but were easily patched to exclude the known counterexamples. We identify more serious flaws.)

Initial results on the maximising problem appear in [5]. The method of attack is via a certain integral involving rook polynomials. These polynomials are studied in some depth, including their connections with Laguerre polynomials. An important step is to relate properties of the roots of the rook polynomials to the number of short cycles in an associated bipartite graph. On this issue we ask the following question. If $G$ is a $k$-regular bipartite graph on $2 n$ vertices $(n>2 k)$ such that $G$ maximises the number of 4-cycles among all such graphs, does $G$ always contain a copy of $K_{k, k}$ ? It is shown

Received 17th August, 1998

Thesis submitted to the Australian National University, November 1997. Degree approved, July 1998. Supervisor: Dr Brendan D. McKay.

Copyright Clearance Centre, Inc. Serial-fee code: 0004-9729/99 \$A2.00+0.00. 
how an affirmative answer would completely characterize $G$. The maximising problem for $n-k \ll n$ could then be resolved using a result from [3].

Chapter 3 was the subject of the B.H. Neumann award winning talk at the 1996 meeting of the Australian Mathematical Society [7]. In that talk the long standing Holens-Đoković conjecture $[4,2]$ on the ratio of subpermanent sums was shown to fail, by converting it to a problem on the ratio of perfect matchings to near perfect matchings in a graph. As a result the problem known as 'monotonicity of the permanent' is answered in the negative. This question asked whether for every doubly stochastic matrix $A$ of order $n$, the permanent is a monotone function on the interval joining $A$ to $J_{n}$, the matrix in which every entry is $1 / n$. Despite the disproof of the general case of the Holens-Đoković conjecture, several specialised cases are proved.

In the final chapter, the asymptotic distribution of subsquares in Latin rectangles is considered. We find that almost all Latin squares contain a large number of intercalates (order 2 subsquares). We also show that $N_{\infty}$ squares (Latin squares without proper subsquares) are very rare and then briefly explore a method of creating such squares. The smallest order for which existence of an $N_{\infty}$ square is unknown is raised from 24 to 256 . This last result has been improved in recent work by the author (as yet unpublished).

\section{REFERENCES}

[1] L.M. Brègman, 'Some properties of nonnegative matrices and their permanents', Soviet Math. Dokl. 14 (1973), 945-949.

[2] D.Ž. Đoković, 'On a conjecture by van der Waerden', Mat. Vesnik 19 (1967), 566-569.

[3] C.D. Godsil and B.D. McKay, 'Asymptotic enumeration of Latin rectangles', J. Combin. Theory. Ser. B 48 (1990), 19-44.

[4] F. Holens, Two aspects of doubly stochastic matrices: Permutation matrices and the minimum permanent function, Ph.D. Thesis (University of Manitoba, 1964).

[5] B.D. McKay and I.M. Wanless, 'Maximising the permanent of $(0,1)$-matrices and the number of extensions of Latin rectangles', Electron. J. Combin. 5 (1998), R11.

[6] H. Minc, 'Theory of permanents 1982-1985', Linear and Multilinear Algebra 21 (1987), 109-148.

[7] I.M. Wanless, 'Jerrum's multistorey carpark', Austral. Math. Soc. Gaz. 23 (1996), 193-197.

Department of Mathematics and Statistics

University of Melbourne

Parkville Vic 3052

Australia

e-mail: ianw@ms.unimelb.edu.au 